\title{
Fuzzy Logic Controlled Protocol for WLANs
}

\author{
Chien-Tung Lu Yu-Sun Liu \\ Dept. of Electronic Engineering \\ Nation Taipei Univ. of Technology \\ Taipei, Taiwan 10608, R.O.C.
}

\begin{abstract}
A fuzzy logic controlled protocol that enables each station to tune its contention window based on the online channel sensing results is proposed for the wireless local area networks. Extensive simulations are provided to compare the performance of the proposed protocol with that of the distributed protocol employed in the IEEE 802.11 standard. Simulation results show that the proposed protocol outperforms the standard protocol in channel utilization, probability of collision, and fairness. Moreover, the proposed protocol well behaves in transition conditions.
\end{abstract}

Keywords: Wireless local area networks, MAC protocol, fuzzy logic control

\section{Introduction}

In the IEEE 802.11 standard [1], [2] for wireless local area networks (WLANs), the basic access method for the medium access control (MAC) layer is the distributed coordination function (DCF). The DCF protocol is based on the carrier-sense multiple access/collision avoidance (CSMA/CA) protocol with the binary exponential backoff scheme, in which random backoff procedure is used to spread the access times of the stations and thus, avoid collisions. References [3] and [4] have shown that the IEEE 802.11 DCF protocol can operate very far from the theoretical throughput limit and proposed adaptive contention window protocols which significantly improve the protocol capacity. In the adaptive protocols, each station dynamically selects the contention window based on the estimate of the number of stations in a WLAN. However, these adaptive protocols are derived under the assumption that all stations always have data frames ready for transmission and, thus, may not be suitable for WLANs with burst stations, which are most common in practice. In this paper, we proposed a fuzzy logic controlled protocol. In the protocol, the network traffic is estimated based on on-line channel sensing results, and the estimated network traffic then serves as a basis for tuning the size of the backoff window. Simulation results are provided to evaluate the performance (including channel utilization, fairness, and responsiveness) of this protocol.

The outline of this paper is as follows. Section 2 describes the standard DCF protocol of WLANs. Section 3 presents the proposed fuzzy logic controlled protocol. The performances of the standard DCF protocol and the proposed protocol are evaluated in Section 4. Concluding remarks can be found in Section 5 .

\section{Standard DCF Protocol}

The standard DCF protocol for WLANs is based on the CSMA/CA protocol. Before a station initiates a new transmission, the station senses the channel to determine whether another station is transmitting. If the medium is found to be idle for an interval that exceeds the distributed inter-frame space (DIFS) period, the station begins its transmission; otherwise, the station monitors the channel until the channel is sensed idle for the duration of a DIFS period and then initials the backoff procedure. At the beginning of a backoff procedure, the station sets its backoff timer to a random backoff time. The backoff timer is decreased as long as the channel is sensed idle, stopped when a transmission is detected on the channel, and reactivated when the channel is sensed idle again for more than a DIFS period. The data packet is transmitted when the backoff timer reaches zero. Immediate positive acknowledgement is employed to ascertain the successful reception of each packet transmission. Immediately following the successful reception of a data packet, the receiver responds, after a short inter-frame space (SIFS), with an acknowledgement frame (ACK). If a positive acknowledgement is not received by the transmitting station within a specific ACK timeout interval, the transmission is presumed to be failed, and the transmitting station invokes the backoff procedure after the channel is idle for a DIFS period.

The standard DCF protocol adopts a binary exponential backoff scheme. At the beginning of each backoff procedure, the random backoff time is given by 
Table 1 Parameters for input membership functions.

\begin{tabular}{|l|cccc|}
\hline & $a^{i}$ & $b^{i}$ & $c^{i}$ & $d^{i}$ \\
\hline$i=0$ & 0 & 0 & 7.22 & 7.44 \\
\hline$i=1$ & 7.28 & 7.5 & 7.5 & 7.72 \\
\hline$i=2$ & 7.56 & 7.78 & 7.78 & 8 \\
\hline$i=3$ & 7.83 & 8.06 & 8.06 & 8.28 \\
\hline$i=4$ & 8.11 & 8.33 & 1023 & 1023 \\
\hline
\end{tabular}

backoff time $=R \times$ a backoff slot time,

where $R$ is uniformly chosen in the range [0, CW-1]. At the first transmission attempt of a packet, the contention window $\mathrm{CW}$ takes an initial value $\mathrm{CW}_{\text {min }}$, and it is doubled at each retransmission up to $\mathrm{CW}_{\max }$. In the IEEE 802.11a standard, $\mathrm{CW}_{\min }=16$ and $\mathrm{CW}_{\max }=1024$.

\section{Fuzzy Logic Controlled Protocol}

The proposed fuzzy logic controlled protocol is identical to the CSMA/CA protocol described in the previous section except how the size of contention window is determined. Instead of the binary exponential backoff scheme, the fuzzy logic controller at each station tunes the size of contention window based on the estimate of network traffic.

The number of consecutive empty slots between two transmissions (see Fig. 1) is a good indicator for network traffic. A small number of consecutive empty slots between two transmissions indicates heavy traffic and vice versa. The fuzzy logic controller uses two average numbers of consecutive empty slots, $X$ and $Y$, to estimate network traffic. The input variable $X$ with range $\left[0,\left(\mathrm{CW}_{\max }-1\right)\right]$ is the average number of consecutive empty slots over the last 30 packets, and the input variable $Y$ with range $\left[0,\left(\mathrm{CW}_{\max }-1\right)\right]$ is the average number of consecutive empty slots over the last 10 packets. We define the term set of $X$ as $R(X)=\left\{R_{X}^{0}, R_{X}^{1}, R_{X}^{2}, R_{X}^{3}, R_{X}^{4}\right\}$ and $Y$ as $R(Y)=\left\{R_{Y}^{0}, R_{Y}^{1}, R_{Y}^{2}, R_{Y}^{3}, R_{Y}^{4}\right\}$. The input membership functions for $R_{X}^{i}$ and $R_{Y}^{i}$ are

$$
\mu_{X}^{i}(s)=\mu_{Y}^{i}(s)=h\left(s ; a^{i}, b^{i}, c^{i}, d^{i}\right)
$$

where

$$
h(s ; a, b, c, d)=\left\{\begin{array}{cc}
\frac{s-a}{b-a} & a \leq s \leq b \\
1 & b \leq s \leq c \\
\frac{d-s}{d-c} & c \leq s \leq d \\
0 & \text { otherwise }
\end{array}\right.
$$

and $a^{i}, b^{i}, c^{i}$, and $d^{i}$ are listed in Table 1. It follows that $R_{X}^{i}$ and $R_{Y}^{i}$ both indicate more consecutive empty slots as $i$ increases. Note that when $i=4, c^{i}$ and $d^{i}$

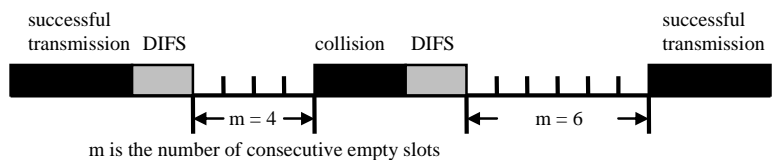

Fig. 1 Diagram of the consecutive empty slots

are equal to $\mathrm{CW}_{\max }-1$.

The output variable $Z$ with range $[0,1]$ is the network traffic indicator. The larger the value of $Z$ is, the heavier the traffic is. We define the term set of $Z$ as $\quad R(z)=\left\{R_{Z}^{0}, R_{Z}^{1}, \ldots, R_{Z}^{8}\right\}$. The membership functions for $R_{Z}^{k}$ are

$$
\mu_{Z}^{k}(z)= \begin{cases}1 & z=1-k / 8 \\ 0 & \text { otherwise. }\end{cases}
$$

Thus, $R_{Z}^{k}$ indicates lighter traffic as $k$ increases. The fuzzy control rules are based on the following observations: if both average numbers of consecutive empty slots, $X$ and $Y$, are small, the traffic is heavy; if both are large, the traffic is light; if one is large and one is small, the traffic is somewhere in the middle. The rule of this fuzzy logic controller contains 25 fuzzy control statements:

$R^{i j}:$ IF $X$ is $R_{X}^{i}$ and $Y$ is $R_{Y}^{j}$, THEN $Z$ is $R_{Z}^{i+j}$

where $i, j \in\{0,1,2,3,4\}$. We use the max-min interference method and the center of area defuzzification method to obtain the network traffic indicator $Z$.

The new $\mathrm{CW}$ value is determined according to the current $\mathrm{CW}$ value as well as the traffic indicator $Z$. If the last transmission is successful,

$$
\mathrm{CW}_{\text {new }}=\max \left\{\mathrm{CW}_{\text {old }} \times f_{\text {succ }}(z), \mathrm{CW}_{\text {min }}\right\} ;
$$

otherwise,

$$
\mathrm{CW}_{\text {new }}=\min \left\{\mathrm{CW}_{\text {old }} \times f_{\text {fail }}(\mathrm{z}), \mathrm{CW}_{\text {max }}\right\},
$$

where $\mathrm{CW}_{\text {new }}$ and $\mathrm{CW}_{\text {old }}$ are the new and the current value of $\mathrm{CW}$, respectively. The figures of the $\mathrm{CW}$ control function, $f_{\text {succ }}(z)$ (and $f_{\text {fail }}(z)$ ), is the union of four line sections which connect five points $(0,0.015)$, (0.3, 0.097), (0.45, 0986), $(0.9,1)$, and $(1,1)$ (respectively, $(0,1),(0.45,51.4),(0.8,60),(0.9,64)$, and $(1,64))$. Note that $f_{\text {succ }}(z)$ and $f_{\text {fail }}(z)$ are both increasing functions of traffic indicator $Z$, since large $Z$ indicates heavy traffic for which large CW is necessary. Moreover, note that $f_{\text {succ }}(z) \leq 1$ and $f_{\text {fail }}(z) \geq 1$. In other word, a station decreases CW after each successful transmission and increases $\mathrm{CW}$, otherwise.

\section{Simulations and Results}


We choose network parameters (e.g. $\mathrm{CW}_{\min }, \mathrm{CW}_{\max }$, DIFS, SIFS, backoff slot time) according to IEEE 802.11a standard [2] at $6 \mathrm{Mbits} / \mathrm{sec}$ transmission rate. The length of frame body in an MAC data frame is given by

$$
\min (2312,\lceil L\rceil) \text { bytes, }
$$

where $L$ is an exponential random variable with mean $\lambda$. All results in this section are obtained under asymptotic condition, which means that every station always has a data packet ready for transmission. Furthermore, all simulations are performed with ideal channel assumption (no transmission errors beside collisions). Finally, we assume that the hiddenterminal problem [5] never occurs, i.e. every station can always hear all others.

\subsection{Channel Utilization}

The utilization of the channel is defined as the fraction of time used by successfully transmitted data packets. The control frames (e.g., ACK), inter-frame periods (e.g., DIFS, SIFS), collisions, and idle slots are not included in the calculation of utilization. The maximum utilization the network can attain, when all stations employ CSMA/CA protocol with same fixed CW value, is called the capacity of CSMA/CA protocol. Note that the optimal value of CW depends on the number of stations. Figure 2 plots the utilizations of the channel for the CSMA/CA protocol and the FLC protocol against the number of transmitting stations for $\lambda=1000$ bytes. We observe that the CSMA/CA protocol often operates very far away from the capacity, especially when there are a large number of stations in the network. This is because in the CSMA/CA protocol, stations always reset the value of $\mathrm{CW}$ to $\mathrm{CW}_{\min }$ after successful transmissions and restart the process of finding the optimal value of $\mathrm{CW}$ at the cost of collisions, which results in serious reduction of channel utilization.

Figure 3 plots the probability of collision for the CSMA/CA and the FLC protocols with $\lambda=1000$ bytes. The collision probability for the CSMA/CA protocol increases drastically with the number of stations.

The FLC protocol significantly enhances the channel utilization by choosing an appropriate CW value based on the estimate of network traffic. If the parameters of the fuzzy logic controller are chosen properly, this $\mathrm{CW}$ value can be very close to the optimal CW value. Therefore, the FLC protocol attains significantly less probability of collision than the CSMA/CA protocol, especially when the network has a large number of stations. The channel utilization of the FLC protocol not only is much larger than that of the CSMA/CA protocol, but also approaches the capacity of CSMA/CA as shown in Fig. 2.

\subsection{Fairness}

In this subsection, we will measure the degree of fairness for the protocols. We use fariness index (FI) to estimate the degree of fairness, which is defined as

$$
F I=\frac{\min _{i \in \omega} U_{i}}{\max _{i \in \omega} U_{i}}
$$

where $U_{i}$ is the number of packets successfully transmitted by station $i$ during a specified time period, and $\omega$ is the set of all stations. Figure 4 plots the fairness index for a time period of 10 seconds with $\lambda=1000$ bytes. From the figure, we observe that the fairness performance of the CSMA/CA protocol deteriorates as the number of stations increases. This is because the CSMA/CA protocol always favors the last succeeding station by resetting its $\mathrm{CW}$ value to $\mathrm{CW}_{\min }$. When channel load is high, a station that has successfully transmitted a packet obtains a statistically much shorter backoff period than other stations. These other stations are at a disadvantage in access to the channel, which results in excessive access delay and severe throughput degradation. In the FLC protocol, the value of $\mathrm{CW}$ of the succeeding station is not reset to $\mathrm{CW}_{\text {min }}$. Therefore, the FLC protocol can mitigate the fairness problem in the CSMA/CA protocol.

\subsection{Responsiveness}

In this section, we investigate the promptness of protocols to re-tune the value of $\mathrm{CW}$ when the number of the transmitting stations changes sharply. Figure 5 plots the utilization of the channel over 2 seconds, which are the average results over 2000 independent experiments. At the beginning of the experiment, the number of transmitting stations $N$ is 0 , and the initial $\mathrm{CW}$ value of each station equals $\mathrm{CW}_{\min }$. At time $0+$, the number of transmitting stations changes from $N=0$ to $N=80$. Again, at time 1.2 seconds, the number of transmitting stations changes from $N=80$ to $N=1$. When the number of transmitting stations changes from 0 to 80 , the FLC protocol reaches the steady-state utilization for $N=80$ much faster than the CSMA/CA protocol does. In the CSMA/CA protocol, each station uses binary exponential backoff mechanism to probe the optimal CW value. When $N=80$, the optimal CW value is large. Thus, when using the CSMA/CA protocol, it takes a large number of collisions and consequently a long time to reach the optimal value of CW. In the FLC protocol, each station adjusts the value of $\mathrm{CW}$ according to the traffic estimation at that time. Therefore, the FLC protocol suffers less collisions than the CSMA/CA protocol before reaching steady-state. When the number of transmitting stations changes from 80 to 1 at time 1.2 
seconds, the CSMA/CA protocol reaches the steadystate utilization for $N=1$ much faster than the FLC protocol. This is because after every successful transmission, the CSMA/CA protocol resets the CW value to $\mathrm{CW}_{\min }$, which is close to the optimal $\mathrm{CW}$ for $N=1$. In the FLC protocol, the average numbers of consecutive empty slots, $X$ and $Y$, have memory which slows down the reaction of the Fuzzy logic controller. However, it takes less than 0.1 second for the FLC protocol to reach steady-state, during which the utilization is high. We conclude that the behavior of the FLC protocol in transition conditions is reasonably good.

\section{Conclusions}

In this paper, we propose a distributed random access protocol for WLANs. In the proposed protocol, each transmitter employs a fuzzy logic controller to tune the $\mathrm{CW}$ value. Extensive simulations are provided to compare the performance of the proposed FLC protocol with that of the CSMA/CA protocols employed in the IEEE 802.11 standard. We find that the proposed FLC protocol significantly increases the channel utilization and reduces the probability of collision. Furthermore, the channel utilization of the FLC protocol approaches the capacity of the CSMA/CA protocol. In addition, the FLC protocol also relieves the fairness problem of the CSMA/CA protocol and well behaves in transition conditions. Thus, we conclude that the proposed FLC protocol is a great improvement of the CSMA/CA protocol for WLANs.

\section{References}

[1] IEEE 802.11, "Wireless LAN medium access control (MAC) and Physical Layer (PHY) specifications,” Aug. 1999.

[2] IEEE 802.11, "Wireless LAN medium access control (MAC) and Physical Layer (PHY) specifications: High-speed Physical Layer in the 5GHz Band,” Sept. 1999.

[3] F. Cali, M. Conti, and E. Gregori, "IEEE 802.11 protocol: Design and performance evaluation of an adaptive backoff mechanism,” IEEE J. Sel. Areas Commun., vol. 18, no. 9, pp. 1774-1786, Sept. 2000.

[4] F. Cali, M. Conti, and E. Gregori, "Dynamic tuning of the IEEE 802.11 protocol to achieve a theoretical throughput limit,” IEEE/ACM Trans. Netw., vol. 8, no. 6, pp. 785-799, Dec. 2000.

[5] F. Tobagi and L.Kleinrock, "Packet switching in radio networks, Part II: The hidden terminal problem in carrier sense multiple access and the busy tone solution,” IEEE Trans. Commun., vol. COM-23, no. 12, pp. 1417-1433, Dec. 1975.

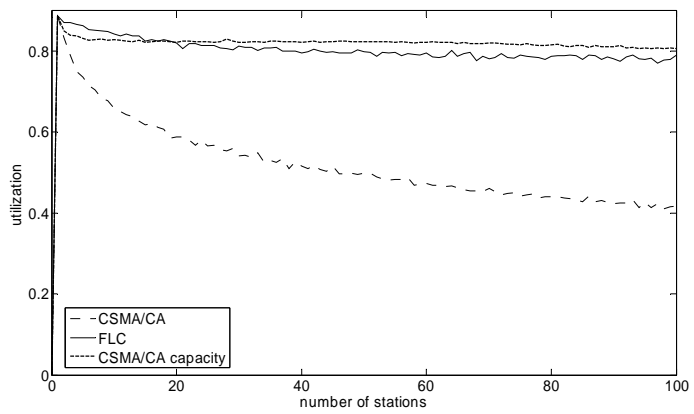

Fig. 2 Channel utilizations for the CSMA/CA and the FLC protocols and capacity of CSMA/CA.

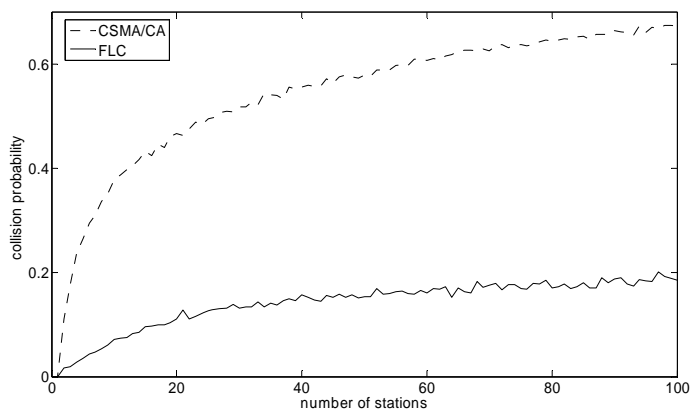

Fig. 3 Probabilities of collision for the CSMA/CA and the FLC protocols.

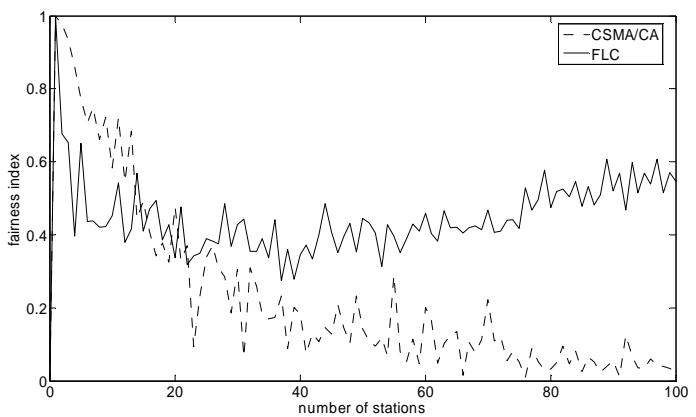

Fig.4 Fairness indexes for the CSMA/CA and the FLC protocols.

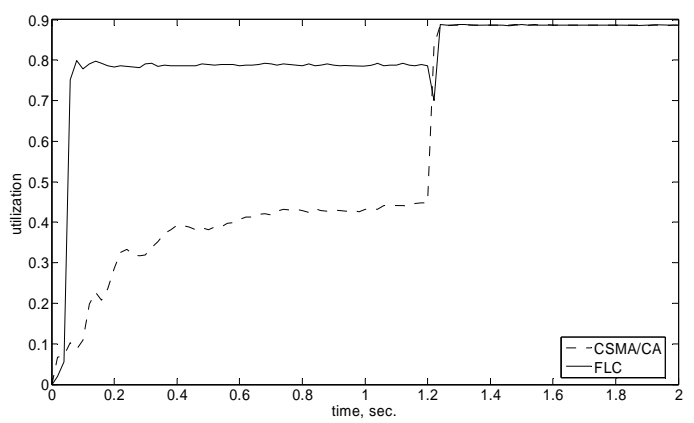

Fig.5 Channel utilizations in transition conditions. 